\title{
News from the San Antonio Breast Cancer Symposium 2009
}

\author{
Chair: Michael Untch \\ Participants: Ingo Bauerfeind ${ }^{\mathrm{b}}$ Christian Jackisch ${ }^{\mathrm{c}}$ Thorsten Kühn ${ }^{\mathrm{d}}$ Volkmar Müller \\ ${ }^{a}$ Clinic for Gynecology, Gynecologic Oncology and Obstetrics, Breast Cancer Center, Academic Hospital of the University Charite Berlin, \\ ${ }^{\mathrm{b}}$ Frauenklinik, Brustzentrum, Klinikum Landshut, \\ ${ }^{\mathrm{c}}$ Department of Obstetrics and Gynecology, \& Breast Cancer Center, Klinikum Offenbach, \\ ${ }^{\mathrm{d}}$ Klinik für Frauenheilkunde und Geburtshilfe, Klinikum Esslingen, \\ ${ }^{\text {e }}$ Department of Gynecology, University Breast Center, University Medical Center Hamburg Eppendorf, Germany
}

\section{Question 1: Risk Adapted, Individualized Therapy Has Been Discussed at ASCO 2009 as well as SABCS 2009. For Breast Cancer Patients this Means the Application of Individual Prognostic Markers, Including Genetic Signatures. Which of these Assays, such as uPA/PAI-1, Oncotype DX or Mammaprint Are Ready for Clinical Practice. Which Do You Use Yourself?}

Bauerfeind: The measurement of uPA/PAI-1 has shown its value in a huge number of patients. uPA/PAI-1 supports us in the decision whether a chemotherapy is indicated in $\mathrm{pT} 1 / 2$ node-negative G2 tumors. ASCO and German AGO recommendations have rated $\mathrm{uPA} / \mathrm{PAI}-1$ as an important adjunct in therapy decision-making. However, there is a certain logistic effort in the routine clinical setting to obtain enough fresh tumor tissue for testing. Furthermore, in many laboratories routine uPA/PAI-1 measurement is not available.

The expected results of the European prospective NNBC 3 trial will support the use of these predictive markers in finding adequate chemotherapeutic protocols. The identification of genetic signatures has not only shown different breast cancer subtypes with probably different clinical behavior but also laid a strong fundament as new prognostic markers. The retrospective correlation of genetic subtypes with clinical outcomes in recent trials has shown promising and challenging results. But as long as no prospective trial has confirmed these data, Oncotype DX and Mammaprint should only be used in clinical trials and not offered routinely to patients.

Jackisch: At present it is important to realize that most of these kits are aimed at an individualized risk assessment of a given patient. But there are some caveats in using these tests in routine clinical practice. Concerning the level of evidence it is important to notice that uPA/PAI-1 is the test with a level I evidence and a validation in some randomized clinical trials (RCT; e.g., NNBC-III). Results are still pending. Oncotype DX and Mammaprint are retrospective evaluates suggesting their potential as an additional expensive prognosticator rather than as a new predictor for treatment options.

I use Oncotype DX in some cases outside RCT if the recurrence score might add information on whether chemotherapy should be added to endocrine treatment, as the test was validated in endocrine responsive disease only. Another advantage is that this test requires paraffin embedded tissue and not fresh frozen tissue like Mammaprint does. In any case we prefer the evaluation of these tools in RCT.

Kühn: There is an essential need for a more tailored adjuvant treatment, especially for intermediate-risk patients. UPA/PA-1, Oncotype DX and Mammaprint have shown a strong prognostic power that is comparable to better-thancommon prognostic markers. All assays seem to have predictive potential for certain subsets of patients, although the predictive value for current chemotherapy regimens has not been proven in prospective trials. We use UPA/PA-1 selectively as an additional prognostic marker, when conventional predictive and prognostic tools do not allow a decision on the use of adjuvant chemotherapy (e.g., N0, G2, moderate endocrine responsiveness). There is a clear advantage for uPA/PA-1 compared to Oncotype DX and Mammaprint with regard to costeffectiveness. Genetic signatures seem to have a higher longterm potential to guide individual treatment decisions.

Müller: If fresh frozen tissue is available, we use uPA/ PAI-1 for node-negative patients. A prospective randomized study (NNBC 3) finished recruitment of 4,150 patients last

\section{KARGER}

Fax +497614520714

Information@Karger.de

www.karger.com (c) 2010 S. Karger GmbH, Freiburg

Accessible online at:

www.karger.com/brc 
year comparing this test with clinico-pathologic risk factors. Results for the rate of patients classified into high- and lowrisk groups were presented in San Antonio 2009 (abstract 4040). Oncotype DX is not covered by public health insurance reimbursement in Germany and no prospective data for its use are available. The great advantage of this test is the use of paraffin-embedded tissue which makes testing easier, especially in small tumors.

One aspect that in my opinion needs further validation is the alteration caused by preoperative core needle biopsies which may lead to dramatic changes in gene expression profiles and also PAI-1 expression. The handling of frozen tissue is difficult. I believe that for routine application, a test should work from paraffin-embedded tissue. In a poster presentation session, several interesting aspects of gene expression signatures for risk estimation were presented. For example, an observational analysis of 5 datasets indicated that grade 2 tumors are more likely to exhibit genomic profile risks discordant with clinical risk assessment compared with grade 1 or 3 tumors. Prognostic gene expression profiles were unable to identify a subpopulation of patients with sufficiently low-risk grade 3 tumors to forego chemotherapy (abstract 103). This underlines the essential role of reliable pathologic grading despite sophisticated gene expression analyses. Abstract 102 showed that in patients with lymph node-positive primary breast cancer genomic profiling combined with nodal status predicts disease outcome. Combining genomic profiling (high vs. low risk) using a 70-gene assay with lymph node status (1-3 vs. 4-9 positive nodes) predicts distant metastasis-free survival and breast cancer specific survival. The authors concluded that patients with high-risk genomic profile and 4-9 positive nodes had shortest survival outcomes and may require aggressive treatment strategies.

\section{Question 2: Which Data Presented at San Antonio 2009 (Together with Data from ASCO 2009) Will Alter Your Therapy Decisions in Postmenopausal Patients with Hormone Receptor-Positive Breast Cancer?}

Bauerfeind: In the adjuvant setting Paul Goss presented a sub-analysis of 889 patients from the BIG 1-98 trial who had been premenopausal at diagnosis and had become postmenopausal during tamoxifen treatment. In the placebo group premenopausal patients had a greater risk for disease recurrence. In the verum group premenopausal women had an absolute difference in 4-year disease free survival (DFS) of $10.1 \%$ whereas postmenopausal patients had an absolute difference in 4-year DFS of $3.3 \%$. The comparison of pre- and postmenopausal women showed a reduced hazard ratio (HR) of 0.39 for the premenopausal patients. In a multivariate analysis node-negative women had an absolute $11.5 \%$ benefit, node positive patients showed an absolute benefit of $9.5 \%$. In my opinion the indication for extended hormonal therapy should be discussed not only in high-risk patients but also in hormone receptor-positive premenopausal breast cancer patients after they become postmenopausal. In the metastatic setting there were no results that alter endocrine therapy options. Again it was shown that the combination of endocrine agents like fulvestrant plus anastrozole did not better than anastrozole given as a single agent (FACT trial). No further studies should be focused on this issue.

Jackisch: Endocine responsive early breast cancer (EBC) in postmenopausal women is a subject for aromatase inhibition for 5 years. Still clear evidence is missing on the question if aromatase inhibition upfront is superior to any sequence using tamoxifen and aromatase inhibition. In low-risk cases in the elderly tamoxifen for 5 years might be still an option. If chemotherapy is used in postmenopausal women with endocrine responsive disease my first choice is use of aromatase inhibition. The update of the BIG 1-98 RCT at the SABCS 2009 demonstrated that the inverse sequence (letrozole $\Rightarrow$ tamoxifen) is not inferior to an upfront use of letrozole for 5 years.

Kühn: The data presented at San Antonio 2009 (BIG 1-98, TEAM, IES) underline the important role of aromatase inhibitors (probable survival benefit for letrozole upfront, confirmed survival benefit and carry-over effect for the sequence of tamoxifen (TAM) and aromatase inhibitor (AI), equal efficacy of exemestane (EXE) vs. TAM-EXE). The presented data do not give any new information to favor either the upfront or the sequence strategy. In high-risk patients, the primary use of letrozole appears preferable. Here, the data presented at San Antonio reinforced the existing policy of most breast centers. A retrospective subgroup analysis from MA 17 was interesting and may change our clinical practice in some patients. Premenopausal patients had significant benefit from extended AI therapy (after 5 years of TAM) when they became postmenopausal under therapy.

Müller: Of importance, a subgroup analysis from the MA 17 trial of women who were premenopausal at the time of diagnosis (abstract 13) and became postmenopausal during tamoxifen was presented. A treatment benefit from extended letrozole therapy with significantly longer DFS and OS for both, node-positive and node-negative patients was observed. DFS improved even more in women premenopausal vs. postmenopausal at diagnosis. This finding was maintained even when letrozole was delayed for up to 6 years after the end of tamoxifen treatment. This should lead us to more attention for this group of women after the end of 5 years of tamoxifen treatment. However, better predictors of ovarian status are required to avoid the stimulation of ovarian function by aromatase inhibitors many clinicians are afraid of.

Results presented from a retrospective cohort analysis by the British Columbia Cancer Agency showed high rates 
(40\% !) of nonadherence to adjuvant tamoxifen and/or aromatase inhibitor treatment in a large healthcare database of postmenopausal women (abstract 36). This underlines the need for improved patient information and management of side effects with endocrine treatment. With the results from the TEAM trial, further evidence for the use of aromatase inhibitors in postmenopausal patients was presented (abstract 11). For me it is still unclear whether adjuvant aromatase inhibitors should be used as mono therapy or sequentially after tamoxifen. With the results from the TEAM study that compared exemestane for 5 years vs. 2-3 years of tamoxifen followed by exemestane in 9,779 postmenopausal women and a 5-year follow-up analysis with absolutely no difference in the end points of DFS, time to recurrence and OS, the strategy of sequential therapy seems a treatment option. However, in this trial, a relevant rate of cross-over occurred. This phenomenon is becoming an increasing problem in clinical trials. An oral presentation compared different approaches for the statistical analysis of the BIG 1-98 trial (abstract 16). However, these models are increasingly difficult to understand for clinicians and even for statisticians.

\section{Question 3: What New Findings Concerning Sentinel Node Examination Have You Learned of That Will Affect Your Clinical Decisions?}

Bauerfeind: Sentinel node biopsy (SNB) is well established and recommended in national and international guidelines for patients with clinically node-negative disease. An open question remains the value of SNB before and after neoadjuvant chemotherapy. The German prospective SENTINA trial, in which over 600 patients have been included to date, will be able to answer these open questions. There is still a controversy, however, which patients need a complete axillary dissection in case of microscopically positive sentinel node(s) or after detection of only isolated tumor cells in the sentinel node. The risk of non-sentinel involvement ranges between 10 and $12 \%$ if isolated tumor cells can be detected and rises to $25 \%$ if microscopic metastases are evident. Although many nomograms and clinical prediction models were published, often not all the data points are available intraoperatively and thus they have limited reliability. Therefore the decision on reoperation has to be postponed until the final histopathological results are known. This leads to increased costs and stress on the patient. Blumencranz et al. introduced the intraoperative GeneSearch ${ }^{\mathrm{TM}}$ BLN assay to predict non-sentinel node involvement: sensitivity, specificity, positive and negative predictive values were good, but not good enough to replace frozen section or final pathological examination. Not touched by these results is the overall prognosis of minimal residual disease in the sentinel(s) that is still a matter of debate. Therefore we follow the German 'AGO recommendations' to perform axillary clearance in the case of micrometastases in the sentinel and recommend no further axillary surgery in the case of only isolated tumor cells in the sentinel.

Jackisch: The implementation of this technique is still breathtaking in reducing surgical aggressiveness for axillary clearence in EBC. I am still fascinated by the evaluation of the SLN procedure in the neoadjuvant setting. After the analysis of the SENTINA trial we will realize that the impact of axillary staging has to be reevaluated.

Kühn: SNB is the accepted standard procedure to determine the axillary status in breast cancer. The future objectives are (i) to reduce the rate of patients who undergo axillary clearance as a diagnostic procedure (patients with 'clinically positive nodes', patients who undergo neoadjuvant chemotherapy) and (ii) to define patients who do not require axillary dissection as a therapeutic procedure (patients with a positive SLN).

The results of the MIRROR study confirm our current recommendations to perform axillary dissection in patients with micrometastases in the SLN. The German SENTINA is designed to give more insight in the role of SNB in patients, who undergo neoadjuvant chemotherapy. The study design has been presented at San Antonio 2009. More than 600 patients have entered this study so far.

Müller: Overall, I saw no presentation that will change our clinical practice. Several presentations tried to define predictive factors for the presence of tumor-involved non-sentinel lymph nodes (poster session 1 and poster presentation session 3). However, we currently have no clearly defined situation where we can omit the complete axillary dissection in patients with one positve sentinel node. One presentation from a Dutch patient cohort concluded that the presence of micrometastatic disease in the sentinel node is in itself not an indication for adjuvant systemic therapy since the prognosis was not affected by this finding (abstract 307).

\section{Question 4: Which New Presentations on Imaging Procedures, Especially on MRI of the Breast, Will Influence Your Therapeutical, Especially the Surgical Decisions in the Future?}

Bauerfeind: The increasing use of magnetic resonance imaging (MRI) before primary breast surgery causes an increasing number of mastecomies. M. Morrow reported a mastectomy rate of $52 \%$ with MRI and $38 \%$ without MRI at the Mayo Clinic, a mastectomy rate of $11,6 \%$ with and 5,3\% without MRI at the Netherland Cancer Institute and a mastectomy rate of $38 \%$ with and $25 \%$ without MRI at the Fox Chase Institute. Whether these mastectomies are necessary in terms of survival is doubtful. Many prospective trials to compare breast conserving therapy versus mastectomy have 
shown their significant equivalence. One might hypothesize that all the small tumors that are now revealed by MRI also existed in previous patient collectives but had no impact on survival or local recurrence.

There are well defined indications to perform MRI of the breast in a selected group of breast cancer patients. MRI of the breast should not be routinely performed in women with newly diagnosed breast cancer when mammography and breast sonography will yield reasonable clinical results. But if MRI is performed and additional - although very small - tumors are detected it is not possible to leave these tumors unremoved.

Jackisch: In our hands MRI has only limited implication in the presurgical procedure. It might be more helpful in the high-risk population linked to genetic instability (e.g., BRCA $1 / 2$ mutation carriers), dense breast tissue etc. MRI will be more important in the screening population.

Kühn: I could not identify any new data on imaging procedures, especially MRI, that could affect our current clinical practice. M. Morrow underlined the need for a critical use of preoperative MRI. There are no data so far showing any impact of MRI on disease free or overall survival. In contrast, there are data from a randomized trial, indicating a higher rate of mastectomies in patients who underwent MRI. In my opinion MRI is a helpful tool in selected patients (lobular carcinoma, high breast density in postmenopausal women, monitoring of neoadjuvant therapy). MRI should be avoided as a routine procedure for patients who undergo breast conserving therapy. The use of MRI should be restricted to institutions that perform MRI-guided core needle biopsy and needle localization.

Müller: Researchers from Taiwan and China found that combining mammography and ultrasound led to a higher cancer detection rate in women aged 40-49 (abstract 72). Researchers in Germany assessed the value of semi-annual ultrasound exams in high-risk women suggesting that this ultrasound screening every 6 months in these women was more valuable than yearly mammography (abstract 4004).

\section{Question 5: What New Developments in Targeted Therapies of Breast Cancer Patients Will Find Their Way into Your Daily Practice?}

Bauerfeind: In the adjuvant and metastatic setting trastuzumab remains the therapy of choice in HER2-positive patients without any prior systemic therapy. The oral tyrosine kinase inhibitor lapatinib has shown high efficacy in the metastatic situation in HER2-positive patients. The first line trials included lapatinib with chemotherapy versus chemotherapy only or the combination of lapatinib plus AI versus AI alone.
In both trials the combination showed significant better results in progression free survival (PFS) and clinical benefit rates, however, without significant influence on survival. At the SABCS meeting an update of the EGF 104900 trial was shown in which the dual HER2 blockade with lapatinib plus trastuzumab versus lapitinib alone was tested. An overall survival benefit was seen following the combination treatment in HER2-positive metastatic breast cancer patients whose disease has progressed under trastuzumab with a $26 \%$ reduction in the risk of death $(\mathrm{p}=0.026)$. Survival benefit was seen also in the setting of $52 \%$ of patients assigned to the single agent lapatinib arm undergoing a planned crossover to the combination arm at the time of progression. The combination arm showed an acceptable tolerability profile without an increase in cardiotoxicity. In my opinion this result offers an alternative in refractory HER2-positive metastatic breast cancer to prolong the chemotherapy free interval.

Two trials presented the efficacy of angiogenesis inhibitor bevacizumab in the metastatic setting: the 25-month update of AVADO trial, in which bevacizumab (with $7.5 \mathrm{mg} / \mathrm{kg}$ or $15 \mathrm{mg} / \mathrm{kg}$ ) plus doxetaxel was randomized versus docetaxel plus placebo, confirmed the initial analyses that docetaxel plus $15 \mathrm{mg} / \mathrm{kg}$ bevacizumab improved PFS and overall response (OR). RIBBON 2 is a prospective randomized, double blind placebo controlled phase III trial evaluating the efficacy and safety of bevacizumab in combination with chemotherapy for second line treatment of HER2-negative metastatic breast cancer. The study demonstrates a statistically significant improvement in PFS and OR for the combination of standard second line chemotherapy plus bevacizumab. The data for overall survival were mentioned as immature. My conclusion: the existing data permit to integrate bevacizumab in the first and second line treatment of HER2-negative metastatic breast cancer patients.

In the metastatic setting the double blind randomized phase IIb trial comparing paclitaxel in the combination with sorafenib versus paclitaxel plus placebo did not show a significant advantage for the verum group. However a multinational double blind randomized placebo controlled phase IIb trial testing capecitabine plus sorafenib versus capecitabine plus placebo showed a significant benefit in PFS (6.4 vs. 4.1 months; HR 0.57, $\mathrm{p}=0.0006$ ). The benefit was seen in first line as well as in second line therapy. In HER2-negative pretreated metastatic patients the comparison of sunitinib versus capecitabine had not met the primary endpoint of improved PFS. Motesanib, a small molecule VEGF tyrosine kinase inhibitor, was compared in combination with weekly paclitaxel (P) versus weekly $\mathrm{P}$ plus bevacizumab versus weekly $\mathrm{P}$ plus placebo as first line therapy in HER2-negative metastatic breast cancer. The OR of the $\mathrm{P}+$ placebo was $41.49 \%$, of $\mathrm{P}+$ motesanib was $49.45 \%$ and of $\mathrm{P}+$ bevacizumab was $51.55 \%$ : The differences between motesanib and placebo and between bevacizumab and motesanib were $p=0.31$ and $p=$ 0.75 , respectively. This short review supports the growing im- 
portance of targeted therapies although the 3 latter substances have to find more clearly their targets to account for their efficacy.

Jackisch: Trastuzumab is introduced intro clinical routine practice in the adjuvant setting. In the neoadjuvant setting this drug is not approved yet. The most important issue is still a proper testing on core needle biopsy to predict the use of targeted drugs like trastuzumab and lapatinib in the neoadjuvant setting. In addition the use of bevacizumab in triple negative disease might be of special interest. An issue of major debate is at present if patients with HER2 overexpressing breast cancer $>5 \mathrm{~mm}$ are already candiates for targeted drugs in addition to chemotherapy.

Kühn: The data on new agents, such as multikinase inhibitors (sorafenib, sutinib, motesanib) were disappointing at San Antonio 2009. However, there were new data on established targeted drugs (trastuzumab, bevacizumab, lapatinib), that will quickly find their way into our clinical routine. The RIBBON-2 study is the first phase III trial showing a significant benefit in PFS for the second line use of bevacizumab. According to the EGF 104900 trial, a dual HER2 blockade (trastuzumab + lapatinib) is associated with a better OS than lapatinib alone in patients with metastatic breast cancer (MBC) who relapsed after a taxane-, anthracycline- and trastuzumabcontaining treatment. The ELECTRA trial showed a remarkable improvement in TTP in patients who were treated with letrozole and trastuzumab compared to patients who received letrozole alone ( 3.3 vs 15.2 months).

Müller: In the adjuvant treatment of HER2-positive patients, updated results of the N9831 trial show DFS benefit for concurrent vs. sequential use of trastuzumab combined with paclitaxel following doxorubicin/cyclophosphamide (abstract 80 ). A strong trend for $25 \%$ reduced DFS event risk when initiating trastuzumab concurrently with paclitaxel compared with sequential use was observed. No difference in OS among treatment arms was observed. Based on these findings and a benefit/risk analysis, we will mainly use adjuvant trastuzumab concurrent with initiation of taxane therapy. In the third planned analysis of BCIRG 006 trial, a nonsignificant trend toward better DFS outcomes with an anthracycline-containing trastuzumab regimen vs. a nonanthracycline-containing regimen was observed. Although this was presented in a way that emphasized the disadvantages of anthracycline side effects, the data for me supports our clinical practice of using anthracycline-free regimens only in patients with cardiac risk factors.

\section{Question 6: Which of the Presented Data on Adjuvant and Neoadjuvant Treatment Will Be Applied in Your Clinical Routine?}

Bauerfeind: After the 65-month follow up and after 656 DFS events and 348 deaths the third planned analysis of BCIRG 006 trial was reported by D. Slamon. Compared to antracycline/cyclophosphamide/docetaxel (ACT) without trastuzumab the combination arms antracycline/cyclophosphamide/docetaxel plus trastuzumab (ACTH) and carboplatin/docetaxel plus trastuzumab (TCH) both trastuzumab arms showed a significantly better DFS $(84,81$, and $75 \%$, respectively). The total number of DFS events were 257 in the ACT arm, 185 in the ACTH arm and 214 in the TCH arm. Although there are 29 events less in the ACTH arm the difference between ACTH and TCH is not statistically significant $(\mathrm{p}=0.02)$, but the absolute difference doubled from 14 to 29 events during the second and third analysis. ACTH and TCH resulted in a significantly better overall survival than ACT with 92,91 and $87 \%$ respectively. The numbers of deaths were 141 in the ACT arm, 94 in the ACTH arm and 113 in the TCH arm. Again the results between both trastuzumab arms did not differ significantly. Both trastuzumab arms worked well in node negative and node positive patients. There were 6 acute leukemias in the ACT arm and 1 in each trastuzumab arm. There was no cardiac related death in any treatment group. Chronic heart failure grade 3 and 4 were 7 in the ACT arm, 21 in the ACTH arm, and 4 in the TCH arm. The difference between ACTH and TCH is significant $(p=0.001)$. The therapeutic index of both trastuzumab arms, nothing else than weighing breast cancer risk versus side effects probability, shows 206 DFS events in the ACTH arm and 218 in the TCH arm. To summarize: both regimens seem to be equieffective but the funeral march that was commenced for anthracycline therapy in breast cancer in the year 2005 was not justified.

No striking new neoadjuvant protocols have been reported in San Antonio. The ongoing German neoadjuvant GeparQuinto study implies current topics concerning chemotherapeutic drugs in combination with modern targeted therapies (trastuzumab, lapatinib, Rad 001, bevacizumab) in HER2positive and HER2-negative patients and should be further supported. In this context surgical complication with the use of neoadjuvant bevacizumab were presented at the meeting. This trial compared surgical data from two single arm neoadjuvant trials in triple negative breast cancer. The patients received $4 \times$ presurgical cisplatin every 3 weeks or $4 \times$ cisplatin every 3 weeks plus $3 \times$ bevacizumab every 3 weeks. All patients received further standard chemotherapy after surgery! Breast conserving surgery was achieved in $46 \%$ (cisplatin) vs. 
57\% (cisplatin plus bevacizumab), respectively. No reconstruction was performed in 10 and 14 patients, expander implantation was done in 3 and 6 patients and a TRAM reconstruction was performed in 2 patients per group. The 'all complication rate' was remarkable but not statistically different between both protocols (39 vs. $43 \%$ ) and included common surgical side effects like seromas, hematomas, wound breakdown and abscesses. Although with no statistically significant difference the loss of reconstruction in the cisplatin/bevacizumab trial was disastrous with 4 complete failures from 8 procedures (and none in the cisplatin only trial). From these 4 patients one woman had a silicone implant plus alloderm, the other three women received a saline expander plus alloderm. The authors stated that the use of implants 'could' be problematic in patients who received bevacizumab in a neoadjuvant setting. My opinion is different from this. The number of examined patients is too small to conclude surgical contraindications. No randomization was performed. Cisplatin is not yet well tested in the neoadjuvant setting an its implications for surgical side effects is not well known. From the reconstructive point of view all patients received further chemotherapy after surgery, which may alter wound healing as well. Furthermore, not only were implants used but additional alloderm as well. This double implantation of allogenous material may have additional deleterious effects on fibroblastic activity after chemotherapy. Interestingly, no tram flap loss was reported although one would almost expect a contraproductive action of an antigenetic drug in this method. So the high complication rate in reconstructive surgery after neoadjuvant chemotherapy plus bavacizumab is probably not only the result of the antigenetic effect of one agent but is much more the result of a wrong reconstructive technique in a wrong situation in suboptimally selected patients.

Jackisch: As members of the GBG and AGO-B Study Groups we joined the GeparQuinto trial as well as the ALLTO trial in the adjuvant setting. In the majority of cases we are using neoadjuvant therapy in most of the cases with HER2 overexpression. Oncotype DX is evaluateded in the context of the PLAN B trial of the West-German Study Group.

Kühn: There were no data presented at San Antonio that will change our clinical practice in the use of adjuvant or neoadjuvant chemotherapy. In fact, we do not expect any further improvement of efficacy from new cytotoxic agents, new combinations of these agents or modified schedules. New developments focus on a better patient selection (predictive markers), a reduction of side effects from chemotherapy (e.g., anthracycline-free regimes) and the introduction of targeted agents into the adjuvant setting. The participation in clinical trials appears extremely important in order to improve the adjuvant and neoadjuvant treatment.

Müller: In my opinion, no data were presented that should lead to a change in clinical practice. With the presentation of the results of the ADEBAR study, the authors suggested that EC-Doc provides a feasible and effective alternative option to dose-intensified FEC with a different safety profile in a highrisk breast cancer cohort (abstract 604). Given the high toxicity of the FEC regimen used ('Canadian FEC'), we will not change our practice in node positive patients and taxanebased regimes will remain our standard.

\section{Participants}

Dr. med. Ingo Bauerfeind Frauenklinik, Brustzentrum Klinikum Landshut

Robert-Koch-Str. 1, 84034 Landshut, Germany

Tel. +49 871 698-3219, Fax -3464

ingo.bauerfeind@klinikum-landshut.de

Prof. Dr. med. Christian Jackisch

Klinik für Gynäkologie und Geburtshilfe und Brustzentrum Offenbach Klinikum Offenbach GmbH

Starkenburgring 66, 63069 Offenbach, Germany

Tel. +49 69-8405-3850, Fax -4456

Christian.Jackisch@klinikum-offenbach.de

Prof. Dr. Thorsten Kühn

Klinik für Frauenheilkunde und Geburtshilfe

Klinikum Esslingen

Hirschlandstraße 97, 73730 Esslingen a. N., Germany

Tel. +49 711 3103-3051, Fax -3052

t.kuehn@klinikum-esslingen.de

Priv. Doz. Dr. med. Volkmar Müller

Department of Gynecology, University Breast Center

University Medical Center Hamburg Eppendorf

Martinistrasse 52, 20246 Hamburg, Germany

Tel. +49 40/7410-57606, Fax -40070

vmueller@uke.de 